\title{
Factors associated with quality of care for patients with pancreatic cancer in Australia
}

\author{
Elizabeth A Burmeister', Dianne L O'Connell ${ }^{2}$, Susan J Jordan', David Goldstein ${ }^{3,4}$, Neil Merrett ${ }^{5}$, David K Wyld ${ }^{6,7}$, \\ Vanessa L Beesley ${ }^{\top}$, Helen M Gooden ${ }^{8}$, Monika Janda ${ }^{9}$, Rachel E Neale', on behalf of the Pancreatic Cancer Clinical \\ Working Group (Appendix 1)
}

The known Treating patients with pancreatic cancer is challenging, and socio-demographic factors influence whether patients receive specific treatment forms, such as surgery and chemotherapy.

The new Our composite quality of care score was lower for patients from rural or socially disadvantaged areas; it was higher for patients who first presented to a hospital with a high pancreatic case volume. A higher score was significantly associated with improved survival.

The implications Strategies should be developed which ensure that all patients with pancreatic cancer have the opportunity to receive optimal care from or in conjunction with high pancreatic case volume centres.

$\mathrm{n}$ Australia, pancreatic cancer is the tenth most common cancer, and the fourth leading cause of cancer-related death. ${ }^{1}$ One-year survival is $20 \%$, 5-year survival $6 \% .^{2}$ Treating pancreatic cancer presents distinctive challenges, and requires highly specialised care to achieve optimal outcomes. ${ }^{3}$ Studies in Australia and overseas have shown that fewer patients receive the recommended treatment than expected, ${ }^{4,5}$ that receiving recommended care is inconsistent, ${ }^{6,7}$ and that socio-demographic factors influence the treatment of patients with pancreatic cancer. ${ }^{7,8}$ Treating patients in non-specialised centres appears to at least partly explain these findings. ${ }^{9,10}$

Previous studies have tended to focus on individual types of treatment, such as surgery or chemotherapy. We took a more holistic approach and calculated an overall quality of care score for Australian patients diagnosed with pancreatic cancer. We examined variations in the score associated with patient and health service-related factors, and analysed the relationship between quality of care and survival.

\section{Methods}

This analysis was nested within a population-based study of patterns of care for patients in Australia with pancreatic cancer. Eligible patients were residents of Queensland and New South Wales diagnosed with pancreatic cancer between July 2009 and June 2011. Patients with histological confirmation of pancreatic adenocarcinoma were included, as were patients with presumed pancreatic cancer but without histological or cytological confirmation. Trained research nurses collected information about patient treatment from medical records in public and private facilities. ${ }^{4}$ Patients were excluded from this analysis if they died within one month of diagnosis or clinical staging data were unavailable.

We calculated a quality of care score based on the results of our previously reported Delphi process. ${ }^{11}$ Briefly, clinicians from a

\section{Abstract}

Objectives: To develop a composite score for the quality of care for patients with pancreatic cancer in Australia; to determine whether it was affected by patient and health service-related factors; to assess whether the score and survival were correlated.

Design, participants and setting: We reviewed medical records of patients diagnosed with pancreatic cancer during July 2009 - June 2011 and notified to the Queensland and New South Wales cancer registries.

Design and main outcome measures: Participants were allocated proportional quality of care scores based on indicators derived from a Delphi process, ranging from 0 (lowest) to 1 (highest quality care). Associations between patient and health service-related factors and the score were tested by linear regression, and associations between the score and survival with Kaplan-Meier and Cox proportional hazards methods.

Results: Proportional quality of care scores were assigned to 1571 patients. Scores for patients living in rural areas were significantly lower than for those in major cities (adjusted difference, 11\%; 95\% Cl, 8-13\%); they were higher for patients in the least socio-economically disadvantaged areas ( $v$ most disadvantaged areas: $8 \%$ higher; $95 \% \mathrm{Cl}, 6-11 \%$ ), who were younger, had better Eastern Cooperative Oncology Group performance status, or who first presented to a hospital with a high pancreatic case volume. Higher scores were associated with improved survival; after adjusting for patient-related factors, each 10 percentage point increase in the score reduced the risk of dying by $6 \%$ (hazard ratio, 0.94; 95\% Cl, 0.91-0.97).

Conclusion: Geographic category of residence may influence the quality of care received by patients with pancreatic cancer, and survival could be improved if they received optimal care.

range of specialties involved in care for patients with pancreatic cancer were asked "What is important in the care of patients with pancreatic cancer?" A list of statements was prepared on the basis of a thematic analysis of the responses. The clinicians were asked to score each statement on a scale of 0 ("disagree", "not important") to 10 ("strongly agree", "very important"). The mean score and the coefficient of variation $(\mathrm{CV})$ were calculated for each statement.

\section{Calculating the quality of care score}

We calculated quality of care scores on the basis of the mean Delphi process scores, selecting statements about which there had been reasonable consensus in the Delphi process $(\mathrm{CV} \leq 0.4)$ and when information for assessing whether the item of care had been delivered was available in our database. Eighteen items were included in the analysis (Box 1 ).

For each patient, we calculated a potential score by identifying the items that applied to their clinical situation and summing the 
1 Statements about care for patients with pancreatic cancer deemed to be most important in our Delphi process, patient eligibility criteria, and definition of care received

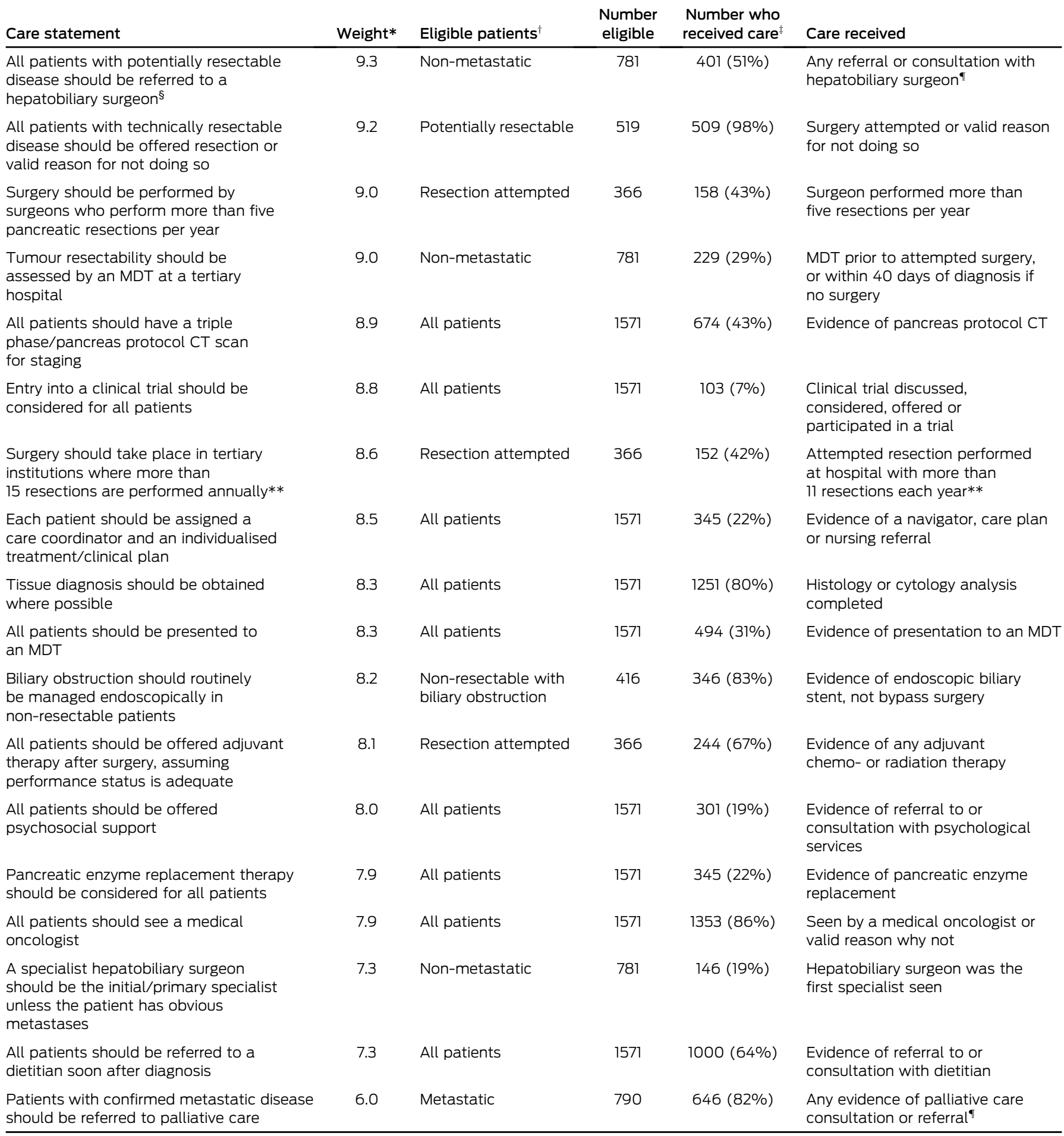

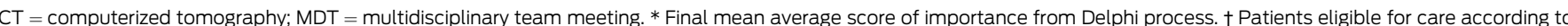

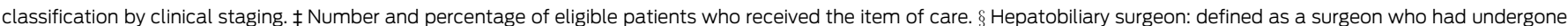

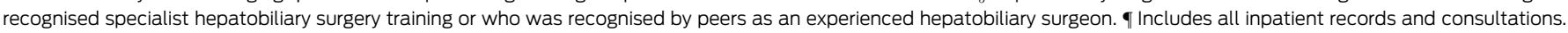

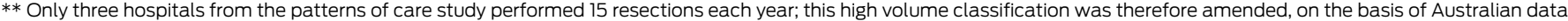
and literature reports, to hospitals where 11 or more resections were performed each year.

mean scores from the Delphi survey for these items. For example, items related to surgical procedures were included only for patients who underwent attempted resection. We then identified items for which there was evidence that the specified care had been delivered and summed their mean Delphi scores as a score for care delivered. The proportional care score was calculated by dividing the care delivered score by the potential score, yielding a value between 0 and 1 . The clinical information that determined eligibility and whether or not care specified by an item was delivered are shown in Box 1. 


\section{Measurement of potential determinants of care}

Patient characteristics assessed included age, sex, Eastern Cooperative Oncology Group (ECOG) performance status, and Charlson comorbidity index. ${ }^{12}$ Based on their area of residence at diagnosis, each person was allocated a socio-economic index for areas (SEIFA) $)^{13}$ score and Accessibility/Remoteness Index of Australia (ARIA +$)^{14}$ category. We grouped the SEIFA scores into quintiles, and collapsed the ARIA into three levels: major city, inner regional, and rural (which included the outer regional, remote and very remote categories).

Tumour-related factors included the stage of the tumour, categorised as potentially resectable or not, and as confined to the pancreas, locally advanced, or metastatic.

Health service-related factors included the type of specialist first seen, and the number of pancreatic cancer presentations (volume) for the facility to which the patient first presented.

\section{Statistical analysis}

The proportions of eligible patients who received each item of care were calculated; the statistical significance of differences between proportions according to socio-economic status and place of residence categories was assessed in $\chi^{2}$ tests.

We used linear regression analyses, with the proportional score as the outcome, to examine variation in the score attributable to patient-, tumour- and health service-related factors. Mean proportional scores for levels of each exposure variable were calculated and $\beta$ coefficients reported (with 95\% confidence intervals [CIs]). The $\beta$ coefficients were interpreted as the difference between the mean score for patients in a particular category and that of patients in the reference category. Multivariable models included age, ECOG performance status, and comorbidity score as factors.

Survival time was calculated from the date of diagnosis until the death of the patient or the date of the final follow-up (February 2014). Patients were grouped in quartiles according to their proportional care scores; Kaplan-Meier graphs were generated and log-rank tests assessed differences in survival according to score quartile. We also performed the analysis with the proportional care score as a continuous variable; we report changes in survival associated with each 10 percentage point increase in score, using Cox proportional hazard models to adjust for patient-related factors and clinical stage. The association between the score and survival was further investigated by calculating adjusted hazard ratios for each care score item separately. Analyses were performed for the entire patient group and separately for patients with or without metastases identified at clinical staging. We used Stata 14 (StataCorp) for all analyses. $P<0.05$ (two-sided) was deemed statistically significant.

\section{Ethics approval}

Access to medical records was approved under the Queensland Public Health Act and the NSW Privacy Act. Ethics approval was obtained from the QIMR Berghofer Medical Research Institute (reference, P1292), the Royal Brisbane and Women's Hospital (on behalf of all public hospitals in Queensland; reference, HREC /10 / QRBW/16), and the NSW Population and Health Services Research Ethics Committee (reference, HREC/10/CIPHS/45).

\section{Results}

A total of 1896 patients were eligible for inclusion in the patterns of care study. We were unable to locate medical records for 33 patients; 259 had died within one month of diagnosis, and staging information was not available for 33, so that 1571 patients (83\%) were included in our analysis, including 867 men (55\%). At clinical staging, 781 patients $(49.7 \%)$ had non-metastatic disease and 790 $(50.3 \%)$ metastatic disease. Most patients lived in major cities (1076, $68 \%) ; 338(22 \%)$ lived in inner regional areas and $157(10 \%)$ in rural areas. Almost three-quarters of patients $(1151,73 \%)$ died within one year of diagnosis. The median survival time was 6 months (11 months for patients without metastases; 4 months for those with metastases).

Younger patients and those with better ECOG performance status had higher care scores than older and less active patients with pancreatic cancer (Appendix 2). ARIA+ category, area level socio-economic status, age, ECOG performance status, institutional pancreatic cancer case volume, and specialist first seen were all factors that significantly influenced the care score (Box 2; Appendix 3). After adjusting for these factors, the care scores for patients living in rural areas were $11 \%$ lower $(95 \% \mathrm{CI}, 8-13 \%)$ than for those living in major cities. The care scores for patients living in more disadvantaged areas were up to $8 \%$ lower (95\% CI, 6-11\%) than for patients living in the least disadvantaged areas. Care score estimates for patients presenting to a low pancreatic cancer case volume hospital (fewer than ten presentations per year) were $13 \%$ lower $(95 \%$ CI, $11-15 \%)$ than for those presenting to hospitals with more than 30 presentations annually. They were higher for patients for whom a hepatobiliary surgeon was the first specialist seen; scores for patients initially seeing a general surgeon were $10 \%$ lower (95\% CI, 8-13\%) (Box 2).

To further investigate the association between ARIA+ category and care score, models were then also adjusted for the pancreatic cancer case volume of the first hospital and specialist seen. The differences in the adjusted mean scores for major cities and rural areas (5\% lower for rural patients; $95 \%$ CI, 3-8\%) and between least and most disadvantaged areas $(6 \%$ lower for most disadvantaged patients; $95 \%$ CI, 3-8\%) were lower in this model.

For patients who had been clinically staged with non-metastatic disease, the factors most strongly associated with lower care scores were being seen initially by a general rather than a hepatobiliary surgeon (17\% lower; $95 \% \mathrm{CI}, 13-21 \%)$, living in a rural area rather than a major city ( $11 \%$ lower; $95 \% \mathrm{CI}, 8-15 \%)$, and being at least 80 years of age ( $v$ aged less than 60 years: $16 \%$ lower; $95 \%$ CI, $13-20 \%$ ). For patients diagnosed with metastatic disease, being seen at a lower volume facility (15\% lower; $95 \%$ CI, $12-17 \%)$ and having a poorer ECOG performance status $(11 \%$ lower; $95 \%$ CI, $7-15 \%$ ) were the factors most strongly associated with quality of care.

Individual items of care were also examined. Less than one-third of patients received some items: $31 \%$ were presented to multidisciplinary teams (MDTs), received psychosocial support (19\%), participated in clinical trials $(7 \%)$, or were first seen by a hepatobiliary surgeon $(19 \%)$. Most eligible patients were offered resection or received a valid reason why they were not $(98 \%)$, had a tissue diagnosis $(80 \%)$, saw a medical oncologist $(86 \%)$, and were referred to palliative care $(82 \%)$ (Box 1$)$. There were significant differences for patients according to their ARIA+ category and area level socio-economic status; for example, 32 patients living in rural areas $(41 \%)$ were referred to a hepatobiliary surgeon, compared with $53 \%$ of patients (290 of 548 ) in metropolitan areas (Appendix 4, Appendix 5).

Patients with scores in the highest quartile of proportional care scores had an estimated median survival time of 8 months, double that for those with scores in the lowest quartile. Median survival 
2 Associations between patient, tumour and health service-related characteristics and proportional care scores for all patients, and for patients with or without evidence of metastases at clinical staging

Adjusted $\beta$ coefficient (95\% confidence interval)*

\begin{tabular}{|c|c|c|c|}
\hline & & & \\
\hline & All patients & Patients without metastases & Patients with metastases \\
\hline Number of patients & 1571 & 781 & 790 \\
\hline \multicolumn{4}{|l|}{ Age group } \\
\hline$<60$ years & Reference & Reference & Reference \\
\hline $60-69$ years & 0.01 ( -0.01 to 0.03$)$ & $0.01(-0.02$ to 0.04$)$ & $0.00(-0.03$ to 0.04$)$ \\
\hline 70-79 years & $-0.05(-0.08$ to -0.03$)$ & $-0.05(-0.08$ to -0.02$)$ & $-0.06(-0.09$ to -0.03$)$ \\
\hline$\geq 80$ years & $-0.13(-0.15$ to -0.10$)$ & $-0.16(-0.20$ to -0.13$)$ & $-0.10(-0.13$ to 0.06$)$ \\
\hline$P$ (overall; trend) & $<0.001 ;<0.001$ & $<0.001 ;<0.001$ & $<0.001 ;<0.001$ \\
\hline \multicolumn{4}{|l|}{ Sex } \\
\hline Women & Reference & Reference & Reference \\
\hline Men & $-0.01(-0.02$ to 0.01$)$ & 0.01 ( -0.01 to 0.03$)$ & $-0.03(-0.05$ to -0.00$)$ \\
\hline$P$ (overall) & 0.34 & 0.40 & 0.03 \\
\hline \multicolumn{4}{|l|}{ Charlson comorbidity score } \\
\hline 0 & Reference & Reference & Reference \\
\hline 1 & $-0.01(-0.03$ to 0.01$)$ & $-0.00(-0.03$ to 0.02$)$ & $-0.01(-0.03$ to 0.02$)$ \\
\hline 2 & $-0.01(-0.03$ to 0.01$)$ & $-0.01(-0.04$ to 0.02$)$ & $-0.01(-0.04$ to 0.02$)$ \\
\hline$P$ (overall; trend) & $0.64 ; 0.38$ & $0.88 ; 0.63$ & $0.89 ; 0.66$ \\
\hline \multicolumn{4}{|l|}{ ECOG performance status } \\
\hline 0 & Reference & Reference & Reference \\
\hline 1 & $-0.01(-0.03$ to 0.01$)$ & $-0.01(-0.04$ to 0.02$)$ & $-0.01(-0.04$ to 0.02$)$ \\
\hline$\geq 2$ & $-0.06(-0.08$ to -0.03$)$ & $-0.06(-0.09$ to -0.03$)$ & $-0.05(-0.08$ to -0.02$)$ \\
\hline Not stated & $-0.09(-0.12$ to -0.06$)$ & $-0.07(-0.11$ to -0.03$)$ & $-0.11(-0.15$ to -0.07$)$ \\
\hline$P$ (overall; trend) & $<0.001 ;<0.001$ & $<0.001 ;<0.001$ & $<0.001 ;<0.001$ \\
\hline \multicolumn{4}{|c|}{ Residence (ARIA+ classification) } \\
\hline Major city & Reference & Reference & Reference \\
\hline Inner regional & $-0.06(-0.08$ to -0.04$)$ & $-0.03(-0.06$ to -0.00$)$ & $-0.08(-0.11$ to -0.05$)$ \\
\hline Rural $^{\dagger}$ & $-0.11(-0.13$ to -0.08$)$ & $-0.11(-0.15$ to -0.08$)$ & $-0.09(-0.13$ to -0.06$)$ \\
\hline$P$ (overall; trend) & $<0.001 ;<0.001$ & $<0.001 ;<0.001$ & $<0.001 ;<0.001$ \\
\hline \multicolumn{4}{|c|}{ Socio-economic status (quintiles) } \\
\hline 1 (least disadvantaged) & Reference & Reference & Reference \\
\hline 2 & $-0.03(-0.06$ to -0.01$)$ & $-0.04(-0.07$ to -0.00$)$ & -0.03 ( -0.07 to 0.01$)$ \\
\hline 3 & $-0.07(-0.10$ to -0.04$)$ & $-0.08(-0.12$ to -0.05$)$ & $-0.06(-0.10$ to -0.02$)$ \\
\hline 4 & $-0.08(-0.11$ to -0.05$)$ & $-0.08(-0.12$ to -0.05$)$ & $-0.08(-0.12$ to -0.04$)$ \\
\hline 5 (most disadvantaged) & $-0.08(-0.11$ to -0.06$)$ & $-0.07(-0.10$ to -0.03$)$ & $-0.10(-0.13$ to -0.06$)$ \\
\hline$P$ (overall; trend) & $<0.001 ;<0.001$ & $<0.001 ;<0.001$ & $<0.001 ;<0.001$ \\
\hline \multicolumn{4}{|l|}{ Clinical stage of disease } \\
\hline Confined to pancreas & Reference & NA & NA \\
\hline Locally advanced & $-0.02(-0.04$ to 0.01$)$ & & \\
\hline Metastatic & $-0.02(-0.04$ to 0.00$)$ & & \\
\hline$P$ (overall; trend) & $0.26 ; 0.14$ & & \\
\hline \multicolumn{4}{|c|}{ Pancreatic cancer case volume of first facility seen } \\
\hline > 30 per year & Reference & Reference & Reference \\
\hline 10-29 per year & $-0.06(-0.08$ to -0.04$)$ & $-0.07(-0.10$ to -0.05$)$ & $-0.04(-0.07$ to -0.02$)$ \\
\hline$<10$ per year & $-0.13(-0.15$ to -0.11$)$ & $-0.10(-0.13$ to -0.07$)$ & $-0.15(-0.17$ to -0.12$)$ \\
\hline$P$ (overall; trend) & $<0.001 ;<0.001$ & $<0.001 ;<0.001$ & $<0.001 ;<0.001$ \\
\hline \multicolumn{4}{|l|}{ First specialist seen } \\
\hline Hepatobiliary surgeon & Reference & Reference & Reference \\
\hline Gastroenterologist & $-0.09(-0.11$ to -0.06$)$ & $-0.12(-0.15$ to -0.09$)$ & $-0.03(-0.07$ to 0.01$)$ \\
\hline General surgeon & $-0.10(-0.13$ to -0.08$)$ & $-0.13(-0.16$ to -0.10$)$ & $-0.05(-0.09$ to -0.01$)$ \\
\hline Other & $-0.14(-0.16$ to -0.11$)$ & $-0.17(-0.21$ to -0.13$)$ & $-0.10(-0.14$ to -0.06$)$ \\
\hline$P$ (overall) & $<0.001$ & $<0.001$ & $<0.001$ \\
\hline
\end{tabular}

ECOG = Eastern Cooperative Oncology Group; NA = not applicable. * Adjusted for age group at diagnosis $(<60,60-69,70-79, \geq 80$ years), ECOG performance status ( $0,1, \geq 2$, not stated), and Charlson comorbidity index score $(0,1, \geq 2)$. † Includes patients in outer regional, remote and very remote areas. 
time for patients with non-metastatic disease in the highest and lowest score quartiles was 14 and 7 months respectively; for those with metastatic disease, it was 5 and 3 months (Box 3).

After adjusting for age, ECOG performance status, comorbidities, and clinical stage of pancreatic disease, each 10 percentage point increase in proportional care score was associated with a statistically significant $6 \%$ reduction in the risk of dying (hazard ratio [HR], 0.94; 95\% CI, 0.91-0.97; Box 4). The reduction was greater for patients who were diagnosed with nonmetastatic disease (adjusted HR, 0.91; 95\% CI, 0.87-0.95) than for those with metastatic disease (adjusted HR, 0.95; 95\% CI, 0.91-0.99).

Individual care score items that were statistically significantly associated with survival included having a diagnostic tissue sample collected (HR, 0.66; 95\% CI, $0.57-0.77)$, being offered adjuvant chemotherapy (HR, $0.43 ; 95 \% \mathrm{CI}, 0.33-0.56)$, being referred to a hepatobiliary surgeon if potentially resectable $(\mathrm{HR}, 0.82$; 95\% CI, 0.69-0.96), being presented to an MDT (HR, 0.86; 95\% CI, 0.77-0.96), being offered psychosocial support (HR, 1.24; 95\% CI, 1.09-1.12), pancreatic enzyme replacement therapy (HR, 0.83; HR, 95\% CI, 0.73-0.94), and, if diagnosed with metastatic disease, referral to palliative care (HR, 1.42; 95\% CI, 1.17-1.74) (Appendix 6).

\section{Discussion}

We found that the quality of care for patients with pancreatic cancer varied according to their age, where they live, and the pancreatic cancer case volume of the hospital to which they first presented. We also found that higher quality of care was associated with improved survival. This association was strongest for patients clinically staged with non-metastatic pancreatic cancer, for whom there is more scope for treatment that can increase survival.

Earlier studies found that receiving surgery, chemotherapy and palliative care was influenced by the age, education, place of residence, ethnic background, and marital status of patients. ${ }^{5,7,15}$ By applying a composite measure of care that included a broad range of factors, we found that age and ECOG performance status influenced its overall quality. While this is unsurprising, it is important to recognise that age alone is not a barrier to high quality care. Our more worrying finding is that quality of care varied according to the geographic classification and the area level socio-economic status of the patient's place of residence. This is at least partly explained by differences in access to specialists and care in high case volume centres, suggesting that interventions which ensure that all patients are managed by high volume teams could improve the quality of care.

Our analysis of individual care items found that the proportion of people receiving care from specialist teams, as recommended, was particularly small: fewer than one-third of patients had been referred to an MDT, only half of potentially resectable patients had been referred to a hepatobiliary surgeon, and referral to a clinical trial was only rarely considered, even though these factors have consistently been found to
3 Kaplan-Meier survival curves for all patients, patients with non-metastatic disease and patients with metastatic disease on clinical staging, by proportional care score (quartiles)

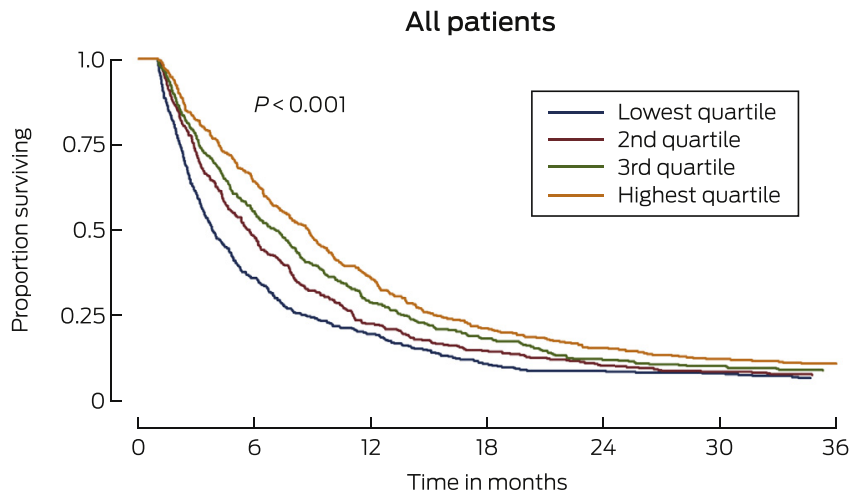

$\begin{array}{llllllll}\text { Number at risk } & & & & & & & \\ \text { Lowest quartile } & 392 & 141 & 77 & 43 & 35 & 32 & 25 \\ \text { 2nd quartile } & 393 & 189 & 89 & 58 & 41 & 34 & 29 \\ \text { 3rd quartile } & 388 & 215 & 112 & 71 & 47 & 39 & 33 \\ \text { Highest quartile } & 398 & 255 & 144 & 85 & 62 & 49 & 38\end{array}$

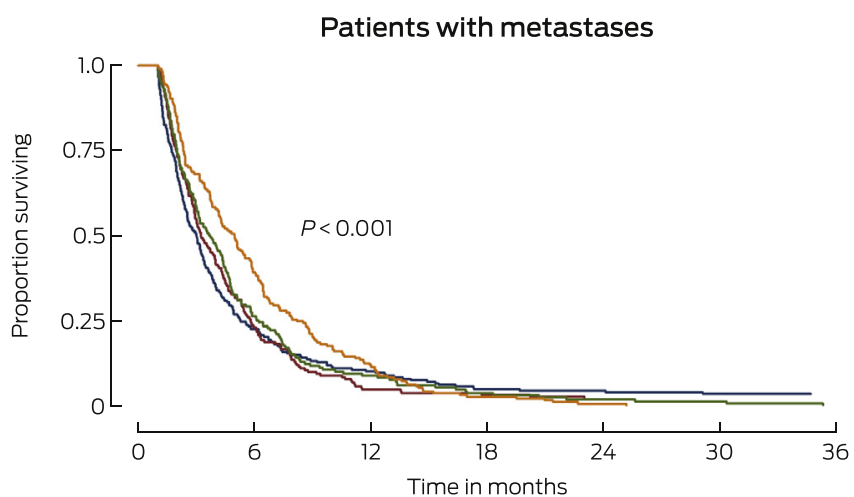

$\begin{array}{llllllll}\text { Number at risk } & & & & & & & \\ \text { Lowest quartile } & 228 & 52 & 24 & 12 & 11 & 5 & 8 \\ \text { 2nd quartile } & 194 & 45 & 10 & 7 & 5 & 5 & 5 \\ \text { 3rd quartile } & 173 & 46 & 16 & 7 & 4 & 3 & 1 \\ \text { Highest quartile } & 195 & 77 & 24 & 6 & 2 & 1 & 1\end{array}$

Patients without metastases

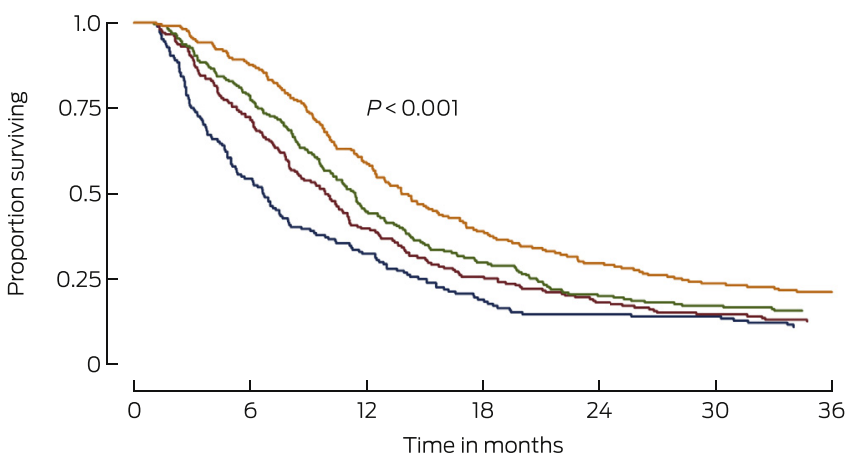

Number at risk

Lowest quartile 2nd quartile

3rd quartile

Highest quartile

$\begin{array}{lllllll}164 & 89 & 53 & 31 & 24 & 23 & 17 \\ 199 & 144 & 79 & 51 & 36 & 29 & 24 \\ 215 & 169 & 96 & 64 & 43 & 36 & 32 \\ 203 & 178 & 120 & 79 & 60 & 48 & 37\end{array}$

* Log-rank test of equality of survivor functions across proportional care score quartiles.

influence the quality of care. ${ }^{9,16,17}$ These aspects of care were particularly poorly delivered to patients living in more rural areas. Distance causes particular challenges in Australia, ${ }^{18-20}$ but they should not be insurmountable; it has been reported, for example, that a multi-level approach (such as telemedicine MDTs and 
4 Association between total care score and survival according to stage of pancreatic cancer at diagnosis

\begin{tabular}{|c|c|c|c|c|c|}
\hline \multirow{2}{*}{\multicolumn{2}{|c|}{ Number of patients }} & \multicolumn{4}{|c|}{ Hazard ratio $(95 \% \mathrm{Cl}) *$} \\
\hline & & Unadjusted & $P$ & Adjusted $^{\dagger}$ & $P$ \\
\hline All patients & 1571 & $0.90(0.87-0.93)$ & $<0.001$ & $0.94(0.91-0.97)$ & $<0.001$ \\
\hline Non-metastatic disease & 781 & $0.87(0.83-0.91)$ & $<0.001$ & $0.91(0.87-0.95)$ & $<0.001$ \\
\hline Metastatic disease & 790 & $0.95(0.91-0.98)$ & 0.006 & $0.95(0.91-0.99)$ & 0.013 \\
\hline
\end{tabular}

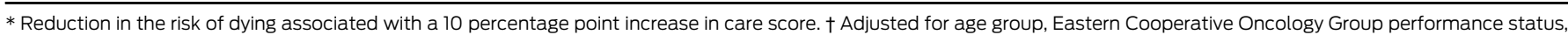
Charlson comorbidity score, and clinical stage.

formalising referral relationships between regional and metropolitan centres) can improve outcomes. ${ }^{21}$

Survival for patients with lower care scores was poorer, consistent with previous reports. ${ }^{22-24}$ This association was stronger for patients diagnosed with non-metastatic disease, for whom there is more scope for influencing survival by ensuring that staging is adequate, that surgery is undertaken in high case volume centres, and that patients have access to adjuvant chemotherapy. For patients with metastatic disease, a focus on quality-of-life indicators is arguably more important; this could be explored in further investigations of care quality.

Some care items were associated with a greater hazard of dying when the care was received, including statements that patients should be "offered psychosocial support", that "patients with metastatic disease should be referred to palliative care", and that "patients with technically resectable disease should be offered resection or a valid reason for no surgery". Receiving psychosocial and palliative care is more likely as the expected survival time shortens, and this probably explains these findings (reverse causation). The care item regarding resection was classed as having been delivered if a valid reason for the resection not being offered had been recorded. This applied to $28 \%$ of patients eligible for resection; the reasons for not attempting surgery included older age, comorbidity, and poor ECOG performance status, each of which were associated with poor survival. When these three care items were all omitted from the care score, the risk of death was $2 \%$ lower for each 10 percentage point increase in care score (data not shown).

Our study was comprehensive, reasonably large, and populationbased, and was also the first Australian investigation to assess the overall quality of care with a single score. Nevertheless, it had some limitations. Firstly, different weights for the care items may have been obtained if another mix of specialists had participated in the Delphi process. Secondly, the Delphi study highlighted the importance of communication between patients and clinicians. This factor cannot be adequately captured in a medical record review and could therefore not be incorporated into our score, but may have influenced decisions regarding care. Thirdly, some patients may have been incorrectly classified as having resectable tumours, which would have affected their eligibility for certain care items and thereby the delivery of appropriate care. Finally, although we controlled for age, ECOG performance status and comorbidities, we may not have completely accounted for confounding patient-related factors.

In conclusion, our population-based study provides evidence that the geographical location of their place of residence, among other factors, influences the quality of care received by Australian patients with pancreatic cancer, and that survival can be improved by delivering optimal care. Systems of care need to be implemented which ensure that equitable treatment is provided for all Australian patients with pancreatic cancer.

\section{Acknowledgements: This investigation was funded by a National Health and Medical Research Council (NHMRC) project grant. Rachel Neale, Susan Jordan and Monika Janda are funded by NHMRC} fellowships. Elizabeth Burmeister is funded by an NHMRC doctoral scholarship.

Competing interests: David Goldstein has received institutional research grants from Amgen, Pfizer, Celgene, Cancer Institute NSW, and Cancer Australia. He has had unremunerated consultancies with Pfizer, Roche and Bayer, and has sat on data and safety monitoring boards for Roche and Sun Biopharma.

Received 12 May 2016, accepted 1 Aug 2016.

(c) 2016 AMPCo Pty Ltd. Produced with Elsevier B.V. All rights reserved.
1 Australian Institute of Health and Welfare. Cancer in Australia: an overview, 2014 (AlHW Cat. No. CAN 88; Cancer Series No. 90). Canberra: AlHW, 2014. http:// www.aihw.gov.au/publication-detail/?id=60129550047 (accessed Aug 2016)

2 Australian Institute of Health and Welfare. Cancer survival and prevalence in Australia: period estimates from 1982 to 2010 (AlHW Cat. No. CAN 65; Cancer Series No. 69). Canberra: AlHW, 2012. http://www.aihw.gov.au/ publication-detail/?id=10737422720 (accessed Aug 2016).

3 Grimshaw JM, Russell IT. Effect of clinical guidelines on medical practice: a systematic review of rigorous evaluations. Lancet 1993; 342: 1317-1322.

4 Burmeister EA, O'Connell DL, Beesley VL, et al. Describing patterns of care in pancreatic cancer: a population-based study. Pancreas 2015; 44: 1259-1265.

5 Sharp L, Carsin AE, Cronin-Fenton DP, et al. Is there under-treatment of pancreatic cancer? Evidence from a population-based study in Ireland. Eur I Cancer 2009; 45: 1450-1459.

6 Bilimoria KY, Bentrem DJ, Lillemoe KD, et al. Assessment of pancreatic cancer care in the United States based on formally developed quality indicators. J Natl Cancer Inst 2009; 101: 848-859.

7 Abraham A, Al-Refaie WB, Parsons HM, et al. Disparities in pancreas cancer care. Ann Surg Oncol 2013; 20: 2078-2087.

8 He W, Zhao H, Chan W, et al. Underuse of surgical resection among elderly patients with early-stage pancreatic cancer. Surgery 2015; 158: 1226-1234.

9 Asare EA, Washington MK, Gress DM, et al. Improving the quality of cancer staging. CA Cancer J Clin 2015; 65: 261-263.

10 Waterhouse M, Burmeister E, O'Connell D, et al. Determinants of outcomes following resection for pancreatic cancer: a population-based study. J Gastrointest Surg 2016; 20:1471-1481.
11 Burmeister EA, Jordan SJ, O'Connell DL, et al. Using a Delphi process to determine optimal care for patients with pancreatic cancer. Asia Pac J Clin Oncol 2016; 12: 105-114

12 Charlson ME, Pompei P, Ales KL, et al. A new method of classifying prognostic comorbidity in longitudinal studies: development and validation. J Chronic Dis 1987 40: 373-383.

13 Australian Bureau of Statistics. Socio-Economic Indexes for Areas [website]. Updated Sept 2013. http://www. abs.gov.au/websitedbs/censushome.nsf/home/seifa (accessed Aug 2016).

14 Australian Population and Migration Research Centre. ARIA and accessibility. Accessibility/Remoteness Index of Australia - ARIA + (2011) [website]. http://www. spatialonline.com.au/ARIA 2011/default.aspx (accessed July 2016).

15 Bilimoria KY, Bentrem DJ, Ko CY, et al. Multimodality therapy for pancreatic cancer in the US: utilization, 
outcomes, and the effect of hospital volume. Cancer 2007; 110: 1227-1234.

16 European Partnership Action Against Cancer consensus group. Policy statement on multidisciplinary cancer care. Eur J Cancer 2014; 50: 475-480.

17 Department of Health and Human Services (Victoria). Optimal care pathway for people with pancreatic cancer. Melbourne: DHHS, 2015. http://www.cancer.org.au/ content/ocp/health/Optimal_cancer_care_for_people with_pancreatic_cancer.pdf (accessed Aug 2016).

18 Martin HL, Ohara K, Chin W, et al. Cancer services in Western Australia: a comparison of regional outcomes with metropolitan Perth. Aust J Rural Health 2015; 23: 302-308.

19 Coory MD, Ho T, Jordan SJ. Australia is continuing to make progress against cancer, but the regional and remote disadvantage remains. Med J Aust 2013; 199: 605-608. https://www.mja.com.au/journal/2013/199/9/ australia-continuing-make-progress-against-cancerregional-and-remote

20 Australian Institute of Health and Welfare. Mortality inequalities in Australia 2009-2011 (Bulletin 124). Aug 2014. http://www.aihw.gov.au/publication-detail/?id= 60129548021 (accessed Aug 2016).
21 Langer B, Stern $\mathrm{H}$. An integrated system-wide strategy for quality improvement in cancer surgery. $\mathrm{Br} J$ Surg 2007; 94: 3-5

22 Coit DG. NCCN guidelines and quality cancer care: where have we come from, and where should we be going? I Natl Compr Canc Netw 2016; 14: 373-377.

23 Cheung R. Racial and social economic factors impact on the cause specific survival of pancreatic cancer: a SEER survey. Asian Pac J Cancer Prev 2013; 14: 159-163.

24 Glimelius B, Hoffman K, Sjoden PO, et al. Chemotherapy improves survival and quality of life in advanced pancreatic and biliary cancer. Ann Oncol 1996; 7: 593-600. 